\title{
A Brief Survey on 5G Wireless Mobile Network
}

\author{
Marwan A. Al-Namari ${ }^{1,2}$ \\ ${ }^{1}$ Faculty of Computer Science and Information Technology, \\ University of Malaya, \\ 50603 Kuala Lumpur, Malaysia \\ ${ }^{2}$ College of Computer at Al-Gunfudah, \\ Umm Al-Qura University, \\ Saudi Arabia
}

\begin{abstract}
The new upcoming technology of the fifth generation wireless mobile network is advertised as lightning speed internet, everywhere, for everything, for everyone in the nearest future. There are a lot of efforts and research carrying on many aspects, e.g. millimetre wave $(\mathrm{mmW})$ radio transmission, massive multiple input and multiple output (Massive-MIMO) new antenna technology, the promising technique of SDN architecture, Internet of Thing (IoT) and many more. In this brief survey, we highlight some of the most recent developments towards the 5G mobile network.
\end{abstract}

Keywords-5G; millimetre wave (mmW); Internet of Thing (IoT); SDN; massive multiple input and multiple output (MassiveMIMO)

\section{INTRODUCTION}

The fifth generation network $(5 \mathrm{G})$ is coming sooner than we expect, some say by 2020 [1], which is expected to have a speed exceeding the $1 \mathrm{Gbit} / \mathrm{s}$ [2]. There has been a great interest in the research of the $5 \mathrm{G}$ future technology, so 700 million euro from the public fund has been committed only for this research over seven years [3]. Many aspects could play an important role in forming the $5 \mathrm{G}$ network, in [4] they concentrated on five elements: millimetre wave $(\mathrm{mmW})$, massive multiple input and multiple output (massive -MIMO), device-centric architectures, smarter devices, and native support for machine-to-machine (M2M). The authors in [2] emphasized five things as a challenge for 5G: Heterogeneous Networks (HetNets), Software Defined Cellular Networks (SDN), M-MIMO and 3D MIMO, M2M Communications and other technologies. In [5] the authors talked about four generic elements which could form the 5G era: Big Data Analytics (Big Data), Cloud Computing (Cloud), Internet of Things (IoT), and SDN. In [1] the authors discussed in detail about many aspects related to the upcoming 5G network: Engineering Requirements for $5 \mathrm{G}$ and the design issues, mmW, M- MIMO, Cloud-Based Networking, SDN, Energy Efficiency, spectrum regulation and standardization for $5 \mathrm{G}$ and many more. Fig. 1 depicts some of the related aspects to the new $5 \mathrm{G}$ mobile networks.

The remainder of the paper is organized as follows. Section 2 presents the evolution of the mobile networks. Section 3 gives an overview of the 5G network architecture. Section 4 explains the Software-Defined network (SDN) architecture. Section 5 describes the cloud Radio Access Network (RAN). Section 6 explains the radio network evaluation. Section 7 explains the advanced air interface. Section 8 presents the

\author{
Ali Mohammed Mansoor, Mohd. Yamani Idna Idris \\ Faculty of Computer Science and Information Technology, \\ University of Malaya, \\ 50603 Kuala Lumpur, \\ Malaysia
}

Heterogeneous Network (HetNets). Section9 explains the big data. Section10 describes the Internet of Things (IoT). Section11 presents Internet of Vehicles (IoV). Section12 explains the Device to Device communication (D2D). Section13 presents the Machine to Machine communication (M2M). Section14 explains the millimetre Wave Mobile Communication (mmW). Section15 describes the MassiveMultiple Input Multiple Output (M-MIMO) antenna system. Section 16 presents briefly some proposed ideas of the 5G Energy Efficiency. Section 17 explains the Healthcare related issues to the $5 \mathrm{G}$ network. Section 18 presents some of the available simulations software for the $5 \mathrm{G}$ mobile network. Section 19 draws some possible directions for future research directions. Finally, Section 20 presents the conclusion.

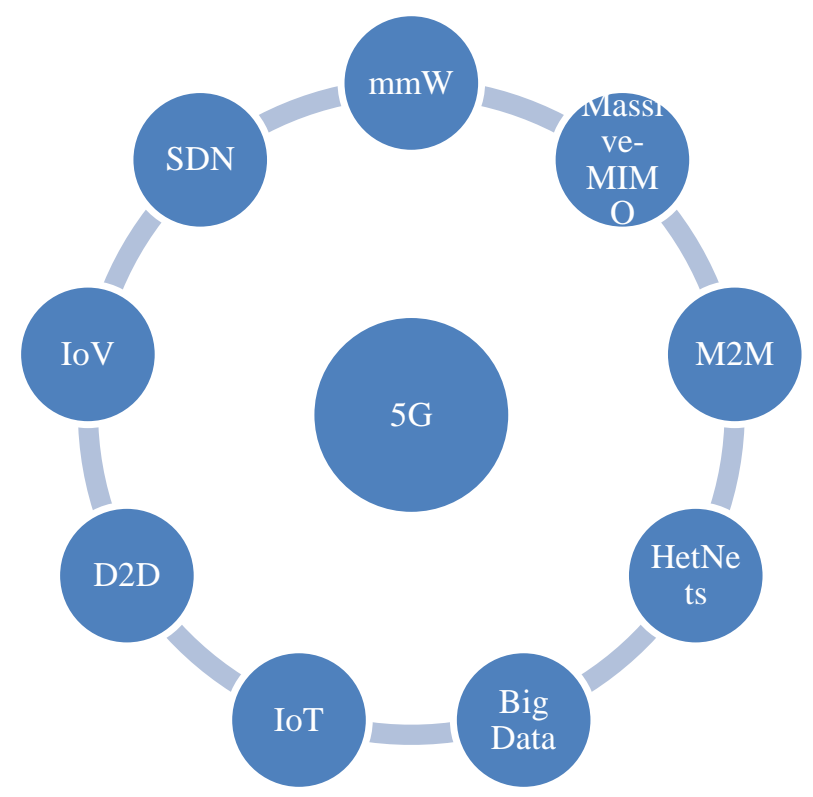

Fig. 1. Some aspects related to the new 5G mobile networks.

\section{Mobile Networks Evolution}

It seems that telecommunication technology advances every decade or so, as $1 \mathrm{G}$ started around 1980, 2G by 1992, 3G by 2001, 4G or Long-Term Evolution (LTE) by 2011 and the new 5G network expectantly by 2020 [6]. 4G nowadays supports $1 \mathrm{Gbits} / \mathrm{s}$ for low mobility and $100 \mathrm{Mbit} / \mathrm{s}$ for high mobility. For the new upcoming $5 \mathrm{G}$ they estimate $10 \mathrm{Gbits} / \mathrm{s}$ for low mobility and 1Gbit/s for high mobility [7]. The latency in $4 \mathrm{G}$ is $15 \mathrm{~ms}$ while in $5 \mathrm{G}$ it is expected to be $1 \mathrm{~ms}$ or so [1]. 
So how could all of that happen? The answer lies in the new architecture of the $5 \mathrm{G}$ network.

\section{5G NETWORK ARCHITECTURE}

The architecture of the new network will be changed, many aspects will try to utilize and get the most out of the existing technology and add new ones to form much faster network capable to deliver the rich content of the multimedia (HD-4K [High Definition] streaming/none streaming video and Hi-Res [High Resolution] images) and the data flood produced from mobile phones and social media apps. One proposed idea is software-defined network architecture. The 5G mobile network needs to deal with some of the challenges facing the $4 \mathrm{G}$ network nowadays such as high energy consumption, spectrum crisis, bad interconnectivity, poor coverage, flexibility, and poor Quality of Service (QoS) [8].

\section{SOFTWARE-DEFINED NETWORK (SDN)}

In [9] the author describes SDN as a promising technique for 5G. SDN and Network Function Virtualization (NFV) have been applied to the cloud in data centres to enable load balance [5]. Furthermore, SDN will play a big role in the design of 5G network as it will be flexible, open and will not be based on switching and routing as the traditional network [9]. Many proposed SDN architectures systems summarized as follows:

- SDN controller that adjusts the bandwidth dynamically as proposed by [10] for each radio access point (RAP) in the network the bandwidth will be dynamically adjusted to the baseband unit (BBU), the SDN controller core consists of two parts: unified control entity (UCE) and unified data gateway (UDW) each part plays a different role in the proposed system, as a whole the SDN controller of the proposed system is providing the flexibility management for the system.

- A new SDN architecture is called SoftAir was proposed by [11]. In this system, the main themes are flexibility and scalability via cloud and virtualization, the data flow is optimized and managed via software-defined switches (SD-switches) and software-defined base station (SD-BSs) in the RAN.

- A new multi-tiered cloud controller SDN architecture, Software Defined Wireless Network (SDWN) proposed by [12] is based on service-oriented and user-centric architecture. The 5G heterogeneous radio access would efficiently improve by decreasing traffic, another advantage of the proposed system is controlling of the quality of user experience (QoE) by varying latency, jitter and throughput to reduce the end-to-end delay to few millisecond to satisfy some future applications.

- Open-source OpenAirInterface (OAI) SDN architecture proposed by [13] is based on two existing technology components used in the LTE architecture: the Evolved Universal Terrestrial Radio Access Network(EUTRAN) and Evolved Packet Core (EPC), the proposed system model is highly realistic as it is based upon a stack of protocols from the network layer all the way to physical layer, the main features of the proposed system are that it is a real-time open-source software implementation of the existing $4 \mathrm{G}$ network model (i.e. 3GPP LTE standard) and it could be used in-door as well as out-door.

- Cross-layer scheme combining Software-Defined Radio (SDR) and SDN architecture was proposed by [14] to combine the two layers together. We need to create this cross-layer to exchange information between them to achieve the best performance of the $5 \mathrm{G}$ mobile network, the proposed system suggests the idea of spectrum reuse to decrease the flow of the traffic in certain frequencies, all of these administration rules need to be governed by the cross-layer controller.

- An intelligent way to deliver data flow SDN architecture was proposed by [15]. This system is based on the SDN controller and how it could analyse the network conditions such as packet loss rate and throughput of the traffic data flow. Based on that it could find the bottleneck and try to solve congestion after calculating the QoS budget. In addition, it can use the network policies to find other alternative routing for the data flow and dynamically update the WAN path routes consequently.

- SoftRAN (Soft Radio Access Network) SDN architecture was proposed by [16], the idea is based on a centralized architecture similar to the SDN architecture by controlling the base stations in one geographical area and uniting them in a big virtual base station, expressed by [11] as attempting to restructure the control plane of RAN in a software-defined way.

\section{Cloud Radio AcCess Network (RAN)}

Cloud Radio Access Network (C-RAN) might solve some problems related to high data rates demands, this technology is based on centralisation and virtualisation [17]. Centralized RAN (C-RAN) is attracting a huge attention as a possible way to centralise computational resources efficiently [18]. In [19], the authors proposed a Dynamic Energy Savings in the 5G Cloud-RAN mobile communication network by combining multiple Base Band Units (BBUs) for signal processing in the centralized BBU pools and using the Wake-on-LAN (WoL) packets to wake up BBUs depending on traffic.

\section{RADIO NETWORK EVALUATION}

The 5G network works towards using $\mathrm{mm}-\mathrm{W}$ signals as a way of transferring high frequency communication. However, there are some limitations for that signal outdoors [6]. When there is no obstacles between the sender and receiver, we have a line of sight (LOS) communication which has a better performance over the non-line of sight (NLOS) communication. If there are obstacles in between the sender and receiver, the signal will reflect and bounce over objects and communication still happens with some loss [20].To cope with the new communication challenges, this proposed technology will provide low cost and less latency by extending the coverage using large beamforming while improving link quality and reducing interference at the end user. The base stations which are used in all previous generations are still a key figure in the coming $5 \mathrm{G}$ network and a new technology 
like mm-W-BS grid proposed by Samsung [21] might be added to them to enhance the performance. An acceptable overlap spectrum occurs from using narrow beams which improves link quality between the base stations and the vast number of end users [22]. The main candidate for the $5 \mathrm{G}$ physical layer is orthogonal frequency-division multiplexing frequency (OFDM). Although, many variants of OFDM are available and have some weakness, tunable OFDM for $5 \mathrm{G}$ is proposed to meet the requirement of the coming $5 \mathrm{G}$ network [23].

\section{ADVANCED AIR INTERFACE}

The antenna topology will change from omni-directional to a directional one [24]. Frequency reuse could be improved by using Spatial Division Multiple Access (SDMA) for beamforming antennas at sender and receiver sides [25]. BS model is governed by many parameters which maximize its performance such as receiver sensitivity, transmit power, antenna height and type, load capacity, operational costs, and capital [26]. In [27], the authors proposed a multiband OFDMA via different mmWave bands, (i.e. the V-band -60 $\mathrm{GHz}$ and the E-band- 70-80 GHz) and the Long Term Evolution (LTE) band for maximizing the overall data rate of the $5 \mathrm{G}$ network by efficient resource allocation. A hybrid analogue and digital beamforming for the large-scale mmWave broadband have been proposed [28] for the single-user MIMO (SU-MIMO) antenna array as it needs to be employed to the transmitter as well as the receiver.

\section{HETEROGENEOUS NETWORK (HETNETS)}

The idea of a big and vast network, which has different classes of base stations: macro, pico and femto [2] by using smaller cells. Here we densify the network by using less power (Green Communication) and improve the coverage of the network [9], by deploying low power small BS to connect with small cells (low transmission power), so the network capacity is improved and coverage is extended [29] to cover indoor and outdoor. If indoor areas have low coverage, the coverage could be enhanced by placing small cells indoor and offloading traffic from macro cells when needed [2]. Unnecessary signalling overhead is generated from Radio Access Technology (RAT) proposed solution to use an efficient multiRAT handover [30]. The architecture of the new 5G network will move from the BS centric to device centric or user centric networks, from small to femto cells deployment which will form the HetNeT. Many technologies are emerging also like cloud-RAN and SDN [31].

\section{BIG DATA}

The new $5 \mathrm{G}$ mobile network is referred to a huge integrated framework of big data [32] passed from multiple sources which needs to be stored and processed. Big data is among the possible technologies which will lead to the $5 \mathrm{G}$ revolution in the nearest future and will aid the traffic for the $5 \mathrm{G}$ users, i.e. Smart cities, VR (virtual reality), IoT, HD videos ,Games, and many more [33]. The architecture of the Big data in 5G is complicated as detailed in the 3GPP standardization process and OIS architecture and could be divided into four layers: application, network, link and physical layer [34].

\section{INTERNET OF THINGS (IOT)}

The next big thing in $5 \mathrm{G}$ is IoT [5] or Internet of Everything (IoE) and sometimes named as Web of Things (WoT)[2]. IoT technology has become embedded in many applications and part of many practical fields like transportation, healthcare, manufacturing, and logistics [35]. Network tasks have become more complex as more and more User Equipment (UE), cars, robots, sensors, and wearables getting smarter and more intelligent, for that different levels, it is required to support reliability, mobility, and spectrum management [36].As everything will have an IP address (IPv6) and will be connected to the Web. Huge number of devices and millions of sensors are connected concurrently to the next 5th generation mobile networks like smart houses and transportation means [37]. There are major threats related to the IoT which need to be considered like security, context sharing, and privacy. Many levels of obstruction and granularity are involved in the HetNet which will be difficult to control [38]. In the future the human centric of the IoT will transform gradually to be Machine to Machine (M2M) platform [39]. Device to Device (D2D) communication and $\mathrm{M} 2 \mathrm{M}$ communication also will be part of the 5G [2]. IoT can be processed online through cloud-base services, for example, Xivelyprovides [40]:

- Open free, source and easy to use graphical user interface (GUI) for the application programming interface.

- Compatibility with many protocols and environments to manage real time sensors and export data in many formats.

- Web-base, real-time visualizing sensor's data graphically, and controlling the sensors remotely.

- Supporting the Original Equipment Manufacturers (OEM) for many manufacturers.

\section{INTERNET OF VEHICLES (IOV)}

$\mathrm{IoV}$ is one of the evaluation application of IoT [41] in which vehicles will be able to communicate between each other and this leads to a smarter transportation with close to null collisions [42]. There are limitations to vehicle-to-vehicle (V2V) communication, while the adjacent car could access the data flow of some sensors and outside viewing cams, the manufacturer of the car could access the full CAN (controller area network) bus sensors and all the viewing cams of the vehicle [43]. Another issue related to the V2V is security, the authors in [44] proposed a new security protection named Multi-factor protection strategy that distinguishes multiple level of privileges of data flow reading from the system controlling. Google with some auto industry are establishing a new protocol related to the vehicles named Open Auto Alliance (OAA) in which they are planning to add new features to Android (the open source platform) to speed up the implementation process of IoV paradigm [45]. A newer standard protocol called IEEE1609 for the Wireless Access in Vehicular Environment (WAVE) has been presented in [46].

The development of self-drive vehicles leads to the Intelligent Transportation System (ITS), many countries have 
started to standardise it such as Europe, USA, and Japan, in order to find a common base of protocols to govern the following challenging issues:

- Privacy, anonymity, and liability

- Congestion control and prioritization of data packets

- Reliability and cross-layering between network and transport layers

- Secure localization

- Addressing and geographical location addressing

- Verification and data-centric trust

- Delay constraints

- Forwarding algorithms

- Risk analysis and management.

Intelligent Transportation System (ITS) may develop further to form Heterogeneous Vehicular NETworks (HetVNETs) which connect with the 5G HetNets[47]. A new standard of Vehicular Ad-hoc networks (VANETs) is forming a new protocol by 2020, which integrates with mobile networks cloud that leads to a safer and smarter transportation system [48].

\section{D2D COMMUNICATION}

Device to Device communication (D2D) is a device centric which originally represents one feature of the new upcoming mobile networks [4] in which indoor small devices close to each other are capable to communicate and share information directly between them and not via the BS [49]. The data traffic of the D2D is growing every year and it is anticipated to increase to 30.6 exabytes monthly by 2020 [50]. By 2021, it is expected the number of machines connected to the net to exceed 28 billion [51]. In [52] the authors proposed for the new $5 \mathrm{G}$ network an ad-hoc D2D network using a group key agreed between the devices which allow them to control routing processes. Many existing technologies will be used in the D2D communication like Near Field Communications (NFC), Ultrawideband (UWB), ZigBee, Bluetooth 4.0, WiFi Direct and LTE Direct, the transmission distance for these wireless standards ranging from less than a meter up to 500 meters [53]. In [54] the authors addressed low latency and how D2D increases the scalability and energy efficiency by controlling the signalling and end to end network communication. Two of the main open issues related to the D2D communication are security and privacy [55]. Therefore, sending and receiving controlling signals and user data in the network of D2D are subject to many kinds of threats including fabrication, manipulation, and eavesdropping [56], in addition, the attacker could hack the system via the broadcast of the wireless communication [57].

\section{M2M COMMUNICATION}

In $5 \mathrm{G}$ network it is expected that $\mathrm{M} 2 \mathrm{M}$ communication to have a native support just like D2D communication [4]. The M2M communication technology becomes smarter and more mobile via the $3 \mathrm{GPP}$ protocols even in the existing LTE network [58]. The M2M data flow is increasing rapidly, i.e. in the mobile network in USA only the M2M traffic volume increased by $250 \%$ in 2011 , and by 2020 it is expected to occupy $45 \%$ of the total traffic of the Internet [59]. The main features about the $\mathrm{M} 2 \mathrm{M}$ in $5 \mathrm{G}$ are self-processing, sharing, and transforming automated data between them with little human effort [60]. The difference between M2M and D2D communication is that $\mathrm{M} 2 \mathrm{M}$ communication connects a vast number of devices, smart meters, sensors and smart grid equipments covering a wide geographical area [49]. The main features of $\mathrm{M} 2 \mathrm{M}$ communication in $5 \mathrm{G}$ mobile network are real time operation, low latency, and high reliability [61].Radio-Frequency Identification (RFID) which comes in many flavours of tag and reader (active, passive or semipassive/active), passive tags do not need battery, whereas active tags are powered by a battery. Also, the second generation (2G-RFID) is a smarter way for the M2M to communicate between them. The process of the communication loops is via five steps: delivering for the mobile code, sensing the object and gathering the information, delivering the information, handling the information, and finally the service response [62]. For the security of the M2M technology using the conventional symmetric and asymmetric cryptographic techniques is enough to secure the communication, but in the case of internal attacks, more advance security methods are need [63].

\section{Millimetre Wave Mobile Communication $(\mathrm{mmW})$}

As the available bandwidth below $6 \mathrm{GHz}$ is limited, engineers start to experiment in the millimetre Wave ( $\mathrm{mmW}$ ) range [2], starting from 3 up to $300 \mathrm{GHz}$ [4]. In [6] the authors did many tests on the $28 \mathrm{GHz}$ and $38 \mathrm{GHz}$ frequencies where they measured loss and gain using different distances. Testing was carried out on many building materials with typical rough and smooth surfaces, i.e. brick and drywalls, clear and tinted glass for their signal reflection and penetration properties. They found $200 \mathrm{~m}$ is the ideal distance with the minimal loss in most conditions.A Google project related to the $5 \mathrm{G}$ millimetre-wave under the name of SkyBender [64] is under testing to deliver very fast internet access (40 times faster than 4G LTE) using multiple-drones powered by solar cells, the testing is taking place at Spaceport America in New Mexico. Before that, Defense Advanced Research Projects Agency (DARPA) had experimented on a similar field, the name of the project is Mobile Hotspots, aiming to help the communication for the military troops in remote areas via deploying multi-drones or Unmanned Aerial Vehicles (UAVs) which provide a communication up to the speed $1 \mathrm{~Gb} / \mathrm{s}$ [65]. The authors in [66] have experimented in merging wire communication with the wireless communication by using low coherence based mmW carrier generation with a dual-colour encoded laser diode to form the hybrid wireless $\mathrm{mmW}$ over Fiber ( $\mathrm{mmWoF}$ ), $12 \mathrm{~Gb} / \mathrm{s}$ is achieved in the newly proposed mmWoF link compared to $36 \mathrm{~Gb} / \mathrm{s}$ in the optical wired band. In [67] the authors proposed a new hybrid architecture for $5 \mathrm{G}$ cellular systems called; RF/millimetre wave, which integrates the RF bands (e.g. $2.4 \mathrm{GHz}$ and $5 \mathrm{GHz}$ ), and mmWave (e.g. spanning the spectrum between $30 \mathrm{GHz}$ to $300 \mathrm{GHz}$ ) interfaces for beamforming and data transfer. 


\section{XV.MAssive- MultiPle InPUT Multiple OutPuT (M- MIMO)}

The next big thing which could change the game in $5 \mathrm{G}$ is the Massive- Multiple Input Multiple Output (M-MIMO). Big communication companies start testing the usability of this technology like SAMSUNG [68], ZTE, and HUAWEI [69] and still are in a race to find the perfect frequency and number of the antenna array with the best beamforming algorithm. In the prototype results, they managed to achieve a data rate of 1.056 $\mathrm{Gb} / \mathrm{s}$ [68] with negligible packet error. Bristol and Lund University with National Instruments (NI) achieved a spectral efficiency of $79.4 \mathrm{bits} / \mathrm{s} / \mathrm{Hz}$ for the first time, and are trying to increase it to $145.6 \mathrm{bits} / \mathrm{s} / \mathrm{Hz}$. All of these results were accomplished via the real-time 128-antenna massive-MIMO testbed, which has been developed by the programmable city initiative- Bristol Is Open (BIO) with National Instruments (NI) and Lund University [70]. Along that they are working in many fields including client localisation, wave front analysis and optimised power control algorithms. To improve the terminal Signal to Interference plus Noise Ratios (SINRs), we need to control the power going to the MIMO system to gain performance in the user side. Using great number of antennas at the massive MIMO-BS side has an effect called channel hardening, which generates new potential for the implementation of efficiency algorithms [71]. The channel hardening effect is a phenomenon which happens when the number of the antennas rises in the MIMO array causing a small percentage of fading. However, in large dimensional signal processing, channel hardening may bring some advantages to the system [72]. If we need to improve the overall massive MIMO system performance, we have to reduce the pilot contamination in Location-based channel-dense deployments estimation by disallowing all mobiles with similar Angle of Arrivals (AoAs) from sharing the same pilot [73]. The conventional massive MIMO is less energy efficient than the hybrid massive MIMO (HMM) system as it is benefited from the newer $5 \mathrm{G}$ architecture technologies including energy harvesting networks, heterogeneous networks, and millimetre wave [74]. Large scaled antenna system (LSAS) or large scale MIMO is a large number of base station configured to serve as one entity and it could serve multiple users in the same time [75]. In [76] the authors discussed the full-dimensional MIMO (FD-MIMO) and how it is explored in the international standardization in 3GPP and how elevation and azimuth get effected from the surrounding environment. Moreover, they addressed the 3-dimensional Multiple-Input Multiple-Output (3D MIMO) which could provide a smart way of implementation, for example, a large number of antennas in a cylindrical array format can serve many users scattered in elevation and azimuth domains.

\section{ENERGY EFFICIENCY}

Controlling power is a major issue in any communication system. Mobile networks worldwide consume $0.5 \%$ of the world's total energy, so one of the main issues should be taken into consideration in the coming $5 \mathrm{G}$ mobile network is reducing the consumption of energy [77]. In [78] a project called 5GrEEn introduces energy-efficient and low latency heterogeneous network (HetNet) architecture. A new idea to save energy proposed in [79] called economical energy efficiency (E3) was based on three things: energy efficiency (EE), spectral efficiency (SE), and cost. It allows analysing the total gain for the $5 \mathrm{G}$ heterogeneous network. Minimising power consumption due to health concerns which requires the power consumption to be reduced in variable transmissions as well as fixed circuits has been discussed in [80].

\section{HEALTHCARE}

The rapid development of sensors leads to a new field in health monitoring [81]. Developing devices with multiple sensors could measure multiple physiological signals at home (just like you are in an Intensive care unit (ICU)) and this technology is available nowadays [82]. Real-time remote patients health monitoring leads to Body Area Networks (BAN) in which big data are collected and processed with a higher data rate and bandwidth, which could be realized in the new 5G network [83]. New range of clinical devices will join the 5G network to form the eHealth and mHealth, In [84], a wide range of wireless, Bluetooth, and NFC devices using the new 6LoWPAN/IEEE 802.15.4 protocol have been addressed.

In [85], an algorithm called Channel State Estimation is designed based on Transmission Power Control (CSE-TPC) for the optimization of the quality of Experience that targets the received signal strength indicator (RSSI) threshold and adjusts the transmission power level. All these factors for the energy efficiency are required for the medical applications in the $5 \mathrm{G}$. Wireless Body Area Networks (WBANs) and Wireless Body Sensor Networks (WBSNs) need this improvement to reduce the consumption of energy to the minimum as mostly the energy for such applications comes from small batteries or embedded rechargeable batteries.

\section{5G SIMULATIONS SOFTWARE}

Many simulation softwares are available online and some are free but others you have to purchase, as mentioned below:

- New York University (NYU) releases 5G mmW simulator; NYUSIM: The open source $5 \mathrm{G}$ channel model simulator software is free as a simulation code (in Matlab) specially for modelling channels from 2 to $73 \mathrm{GHz}$ which is suitable in 3GPP and other standard bodies and academic/industrial simulations [86].

- Fig. 2 shows the graphical user interface (GUI) of the $5 \mathrm{G}$ mmW simulator-NYUSIM.

- A research group under the name of mmMagic supported by SAMSUNG from CSN Group part of Universities of Bristol are working on a simulation of visualising mmWave and beamforming[87]. Fig. 3 depicts the animation of the mmMagic simulation.

- Some Youtube videos are available:

- https://www.youtube.com/watch?v=AaefATDPZMg

- $5 \mathrm{G}$ and MIMO using Wireless InSite

- Remcom has a simulator for MIMO in 5G which handles 3D structures mm-wave and in the near future MIMO with Wireless InSite will be added [88]. Fig. 4 
depicts some of the output results of the InSite Simulator.

- SIRADEL offers simulations since 1994 , and ready to simulate the new $5 \mathrm{G}$, e.g. 3D beamforming or $\mathrm{mmW}$ massive MIMO. They also have a key role in $3 \mathrm{GPP}$ and IEEE standardizations [89]. Fig. 5 depicts the animation of the SIRADEL simulation.

- MATLAB and Simulink to design, test, and prototype $5 \mathrm{G}$ wireless systems

- This simulator which uses MatLab and Simulink are designed for the coming 5G wireless system (design, test and prototype), It includes massive MIMO systems with the beamforming and precoding algorithms for antenna arrays RF system architecture, 5G algorithms, real-time Rapid prototyping, and channel models data for frequencies $>6 \mathrm{Ghz}$ [90]. Fig. 6 shows the MATLAB and Simulink prototype 5G wireless systems.

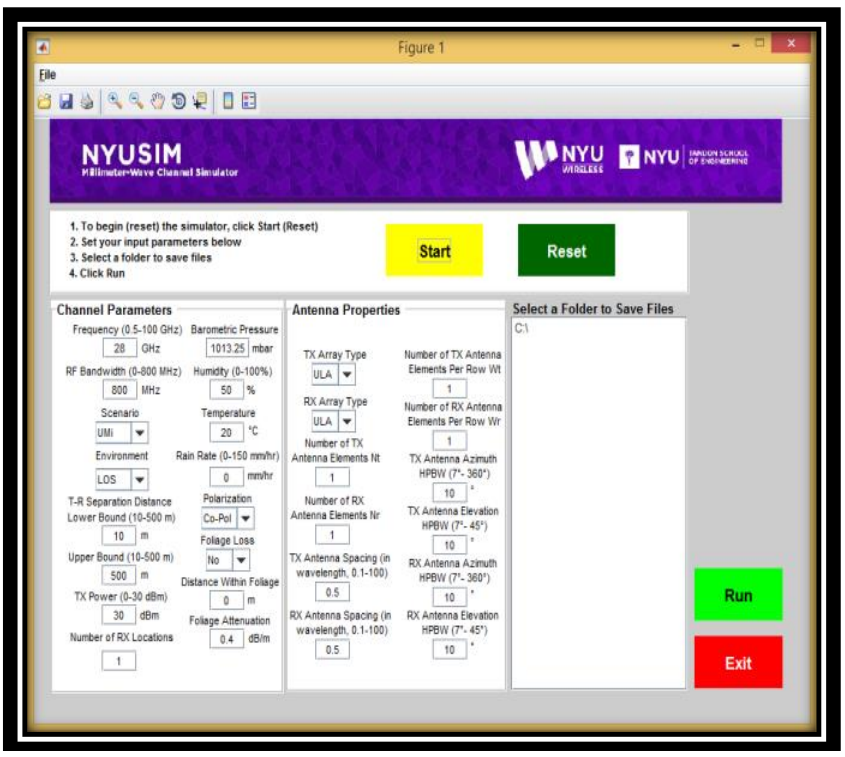

Fig. 2. The GUI of the $5 \mathrm{G} \mathrm{mmW}$ simulator - NYUSIM.

\section{Visualisation and mmWave Network Simulation}
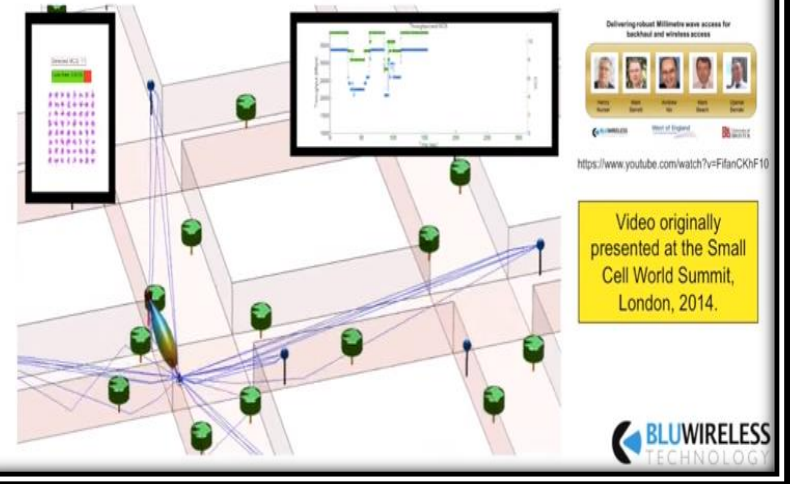

Fig. 3. The animation of the mmMagic simulation.

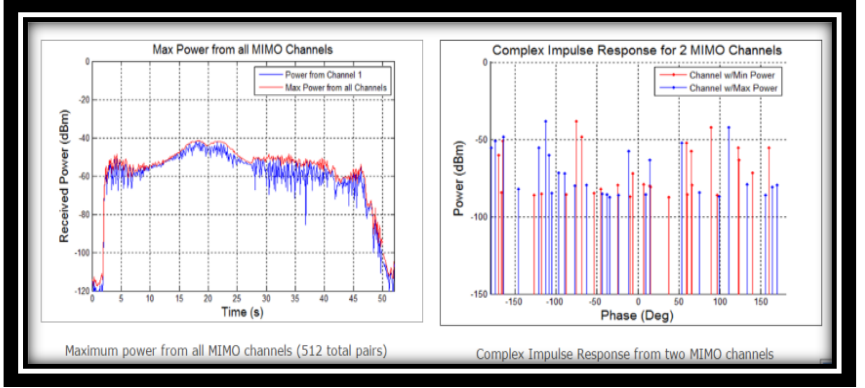

Fig. 4. Some of the output results of the InSite Simulator.

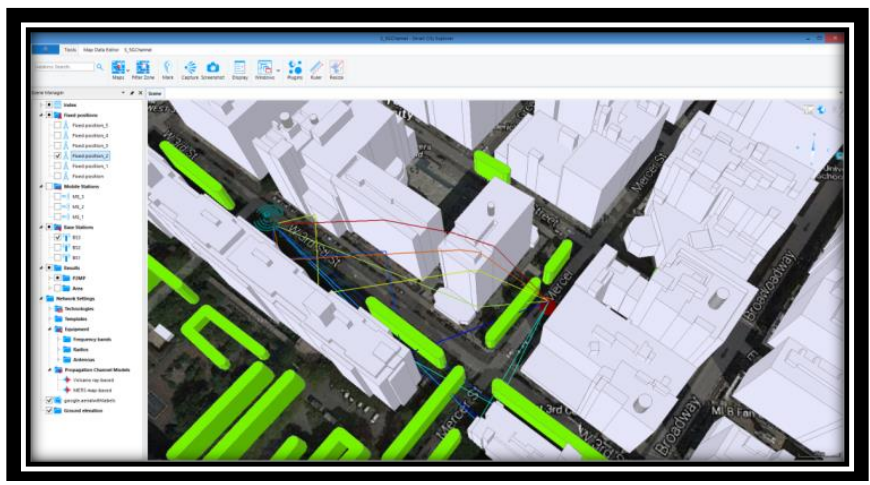

Fig. 5. The animation of the SIRADEL simulation.

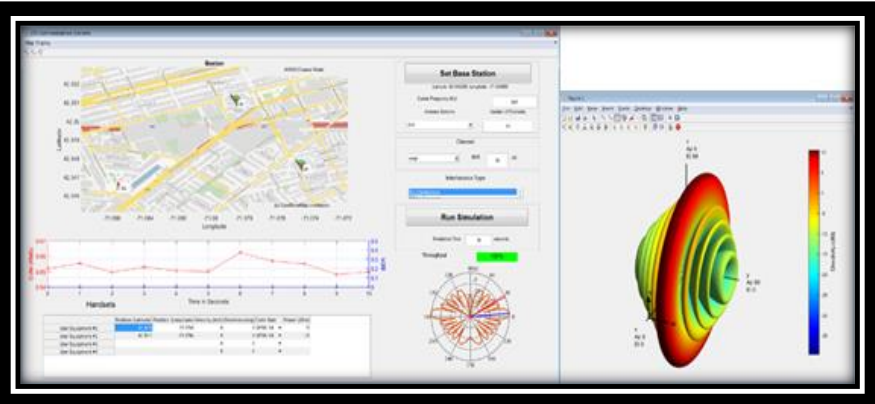

Fig. 6. The MATLAB and Simulink prototype 5G wireless systems.

\section{Future Research Directions}

This survey-as the title depicts- just scratch the surface of the upcoming $5 \mathrm{G}$ mobile networks, many aspects needs to be researched further such as latency and beamforming, backward compatibility with the older generations, multiplexing, power efficiency and green network, 5G applications, standardization, heath issues related to the negative impact of the high frequency waves on humans, connectivity problems and QoS.

\section{XX.CONCLUSION}

In this survey paper, we briefly addressed some evolving technologies related to the $5 \mathrm{G}$ futuristic network. We focused on the main approaches and did not go deeply into the algorithms which govern the power consumption in the $5 \mathrm{G}$ network or any machine learning techniques which could help optimizing the network. This survey could be an adequate entrance knowledge to the new 5G mobile network technology. 


\section{REFERENCES}

[1] Andrews, J.G., et al., What will 5G be? IEEE Journal on Selected Areas in Communications, 2014. 32(6): p. 1065-1082.

[2] Chin, W.H., Z. Fan, and R. Haines, Emerging technologies and research challenges for $5 \mathrm{G}$ wireless networks. IEEE Wireless Communications, 2014. 21(2): p. 106-112.

[3] Bayou, R. European Research towards 5G. in Proceedings of the 21st Annual International Conference on Mobile Computing and Networking. 2015. ACM.

[4] Boccardi, F., et al., Five disruptive technology directions for 5G. IEEE Communications Magazine, 2014. 52(2): p. 74-80.

[5] Lin, B.-S.P., F.J. Lin, and L.-P. Tung, The Roles of 5G Mobile Broadband in the Development of IoT, Big Data, Cloud and SDN. Communications and Network, 2016. 8(01): p. 9.

[6] Rappaport, T.S., et al., Millimeter wave mobile communications for $5 \mathrm{G}$ cellular: It will work! IEEE access, 2013. 1: p. 335-349.

[7] Wang, C.-X., et al., Cellular architecture and key technologies for 5G wireless communication networks. IEEE Communications Magazine, 2014. 52(2): p. 122-130.

[8] Reddy, N.K., A. Hazra, and V. Sukhadeve, A Compact Elliptical Microstrip Patch Antenna for Future 5G Mobile Wireless Communication. Transactions on Engineering \& Applied Sciences, 2017. 1(1): p. 1-4.

[9] Zolanvari, M., SDN for 5G. 2015.

[10] Ma, Z., et al., Key techniques for $5 \mathrm{G}$ wireless communications: network architecture, physical layer, and MAC layer perspectives. Science China Information Sciences, 2015. 58(4): p. 41301-041301 (20).

[11] Akyildiz, I.F., P. Wang, and S.-C. Lin, SoftAir: A software defined networking architecture for $5 \mathrm{G}$ wireless systems. Computer Networks, 2015. 85: p. 1-18.

[12] Sun, G., et al., Software defined wireless network architecture for the next generation mobile communication: Proposal and initial prototype. Journal of Communications, 2014. 9(12): p. 946-953.

[13] Nikaein, N., et al., OpenAirInterface: A flexible platform for 5G research. ACM SIGCOMM Computer Communication Review, 2014. 44(5): p. 33-38.

[14] Cho, H.-H., et al., Integration of SDR and SDN for 5G. IEEE Access, 2014. 2: p. 1196-1204.

[15] Nam, H., D. Calin, and H. Schulzrinne. Intelligent content delivery over wireless via SDN. in Wireless Communications and Networking Conference (WCNC), 2015 IEEE. 2015. IEEE.

[16] Gudipati, A., et al. SoftRAN: Software defined radio access network. in Proceedings of the second ACM SIGCOMM workshop on Hot topics in software defined networking. 2013. ACM.

[17] Checko, A., et al., Cloud RAN for mobile networks-A technology overview. IEEE Communications surveys \& tutorials, 2015. 17(1): p. 405-426.

[18] Rost, P., et al., Cloud technologies for flexible 5G radio access networks. IEEE Communications Magazine, 2014. 52(5): p. 68-76.

[19] Bluemm, C., et al. Dynamic energy savings in Cloud-RAN: An experimental assessment and implementation. in Communications Workshops (ICC Workshops), 2017 IEEE International Conference on. 2017. IEEE.

[20] Rappaport, T.S., et al., Broadband millimeter-wave propagation measurements and models using adaptive-beam antennas for outdoor urban cellular communications. IEEE transactions on antennas and propagation, 2013. 61(4): p. 1850-1859.

[21] Pi, Z. and F. Khan. System design and network architecture for a millimeter-wave mobile broadband (mmb) system. in Sarnoff Symposium, 2011 34th IEEE. 2011. IEEE.

[22] Pi, Z. and F. Khan, An introduction to millimeter-wave mobile broadband systems. IEEE communications magazine, 2011. 49(6).

[23] Aijaz, A., et al., Realizing the tactile internet: Haptic communications over next generation 5G cellular networks. IEEE Wireless Communications, 2017. 24(2): p. 82-89.
[24] Korakis, T., G. Jakllari, and L. Tassiulas, CDR-MAC: A protocol for full exploitation of directional antennas in ad hoc wireless networks. IEEE Transactions on Mobile Computing, 2008. 7(2): p. 145-155.

[25] Bae, J., et al. Architecture and performance evaluation of MmWave based 5G mobile communication system. in Information and Communication Technology Convergence (ICTC), 2014 International Conference on. 2014. IEEE.

[26] Whitaker, R.M. and S. Hurley. Evolution of planning for wireless communication systems. in System Sciences, 2003. Proceedings of the 36th Annual Hawaii International Conference on. 2003. IEEE.

[27] Niknam, S., et al., A Multiband OFDMA Heterogeneous Network for Millimeter Wave 5G Wireless Applications. IEEE Access, 2016. 4: p. 5640-5648.

[28] Sohrabi, F. and W. Yu, Hybrid Analog and Digital Beamforming for mmWave OFDM Large-Scale Antenna Arrays. IEEE Journal on Selected Areas in Communications, 2017.

[29] El-atty, S.M.A. and Z. Gharsseldien. On performance of HetNet with coexisting small cell technology. in Wireless and Mobile Networking Conference (WMNC), 2013 6th Joint IFIP. 2013. IEEE.

[30] Talwar, S., et al. Enabling technologies and architectures for 5G wireless. in Microwave Symposium (IMS), 2014 IEEE MTT-S International. 2014. IEEE.

[31] Agiwal, M., A. Roy, and N. Saxena, Next generation 5G wireless networks: A comprehensive survey. IEEE Communications Surveys \& Tutorials, 2016. 18(3): p. 1617-1655.

[32] Qiu, R.C. and P. Antonik, Big Data for Communications. Smart Grid using Big Data Analytics: A Random Matrix Theory Approach, 2017: p. 525-540.

[33] Gao, Y., et al. Review of wireless big data in 5G: From physical layer to application layer. in Computer and Communications (ICCC), 2016 2nd IEEE International Conference on. 2016. IEEE.

[34] Su, Z., Q. Xu, and Q. Qi, Big data in mobile social networks: a QoEoriented framework. IEEE Network, 2016. 30(1): p. 52-57.

[35] Niyato, D., et al., Practical Perspectives on IoT in 5G Networks: From Theory to Industrial Challenges and Business Opportunities. IEEE Communications Magazine, 2017. 55(2): p. 68-69.

[36] Song, L., et al., Game-theoretic resource allocation methods for deviceto-device communication. IEEE Wireless Communications, 2014. 21(3): p. 136-144.

[37] Center, D.R.D., 5G vision, white paper. 2015, Samsung Electronics Co.

[38] Fortino, G., et al. Integration of agent-based and cloud computing for the smart objects-oriented iot. in Computer Supported Cooperative Work in Design (CSCWD), Proceedings of the 2014 IEEE 18th International Conference on. 2014. IEEE.

[39] Wunder, G., et al., 5GNOW: non-orthogonal, asynchronous waveforms for future mobile applications. IEEE Communications Magazine, 2014. 52(2): p. 97-105.

[40] Doukas, C., Building Internet of Things with the ARDUINO. 2012: CreateSpace Independent Publishing Platform.

[41] Kumar, N., et al., Coalition games for spatio-temporal big data in internet of vehicles environment: A comparative analysis. IEEE Internet of Things Journal, 2015. 2(4): p. 310-320.

[42] Intelligence, G., Understanding 5G: Perspectives on future technologicaladvancements in mobile, White paper. 2014.

[43] Gerla, M., et al. Internet of vehicles: From intelligent grid to autonomous cars and vehicular clouds. in Internet of Things (WF-IoT), 2014 IEEE World Forum on. 2014. IEEE.

[44] Lee, E., et al., Multi-factor authentication and authorization using attribute based identification. UCLA CSD, 2014.

[45] Open Auto Alliance. 2017 10/7/2017]; Available from: https://www.openautoalliance.net/.

[46] Wu, X., et al., Vehicular communications using DSRC: challenges, enhancements, and evolution. IEEE Journal on Selected Areas in Communications, 2013. 31(9): p. 399-408.

[47] Camacho, F., C. Cárdenas, and D. Muñoz, Emerging technologies and research challenges for intelligent transportation systems: 5G, HetNets, 
and SDN. International Journal on Interactive Design and Manufacturing (IJIDeM), 2017: p. 1-9.

[48] Jia, S., et al. Analyzing and relieving the impact of FCD traffic in LTEVANET heterogeneous network. in Telecommunications (ICT), 2014 21st International Conference on. 2014. IEEE.

[49] Asadi, A., Q. Wang, and V. Mancuso, A survey on device-to-device communication in cellular networks. IEEE Communications Surveys \& Tutorials, 2014. 16(4): p. 1801-1819.

[50] Haus, M., et al., Security and Privacy in Device-to-Device (D2D) Communication: A Review. IEEE Communications Surveys \& Tutorials, 2017. 19(2): p. 1054-1079.

[51] Orsino, A., et al., Effects of Heterogeneous Mobility on D2D-and Drone-Assisted Mission-Critical MTC in 5G. IEEE Communications Magazine, 2017. 55(2): p. 79-87.

[52] Jung, Y., E. Festijo, and M. Peradilla. Joint operation of routing control and group key management for 5G ad hoc D2D networks. in Privacy and Security in Mobile Systems (PRISMS), 2014 International Conference on. 2014. IEEE.

[53] Feng, D., et al., Device-to-device communications in cellular networks. IEEE Communications Magazine, 2014. 52(4): p. 49-55.

[54] Yilmaz, O.N., et al. Smart mobility management for D2D communications in 5G networks. in Wireless Communications and Networking Conference Workshops (WCNCW), 2014 IEEE. 2014. IEEE.

[55] Panwar, N., S. Sharma, and A.K. Singh, A survey on 5G: The next generation of mobile communication. Physical Communication, 2016. 18: p. 64-84.

[56] Wang, M. and Z. Yan, A survey on security in D2D communications. Mobile Networks and Applications, 2017. 22(2): p. 195-208.

[57] Gandotra, P., R.K. Jha, and S. Jain, A survey on device-to-device (D2D) communication: Architecture and security issues. Journal of Network and Computer Applications, 2016.

[58] Tuna, G., et al., A survey on information security threats and solutions for Machine to Machine (M2M) communications. Journal of Parallel and Distributed Computing, 2017. 109: p. 142-154.

[59] Al-Fuqaha, A., et al., Internet of things: A survey on enabling technologies, protocols, and applications. IEEE Communications Surveys \& Tutorials, 2015. 17(4): p. 2347-2376.

[60] Zhang, Y., et al., Cognitive machine-to-machine communications: visions and potentials for the smart grid. IEEE network, 2012. 26(3).

[61] Kim, J., et al., M2M Service Platforms: Survey, Issues, and Enabling Technologies. IEEE Communications Surveys and Tutorials, 2014. 16(1): p. 61-76.

[62] Chen, M., Towards smart city: M2M communications with software agent intelligence. Multimedia Tools and Applications, 2013. 67(1): p. 167-178.

[63] Lu, R., et al., GRS: The green, reliability, and security of emerging machine to machine communications. IEEE communications magazine, 2011. 49(4).

[64] Manweiler, J. and M. Baker, From Virtual Football to Fit Freshmen. IEEE Pervasive Computing, 2016. 15(2): p. 86-88.

[65] Remote Troops Closer to Having High-Speed Wireless Networks Mounted on UAVs. 2017 [cited 2017 12/7/2017]; Available from: http://www.darpa.mil/news-events/2014-04-07.

[66] Lin, C.-Y., et al., Millimeter-Wave Carrier Embedded Dual-Color Laser Diode for 5G MMW oF Link. Journal of Lightwave Technology, 2017. 35(12): p. 2409-2420.

[67] Hashemi, M., C.E. Koksal, and N.B. Shroff, Out-of-Band mmWave Beamforming and Communications to Achieve Low Latency and High Energy Efficiency in 5G Systems. arXiv preprint arXiv:1701.06241, 2017.

[68] Roh, W., et al., Millimeter-wave beamforming as an enabling technology for $5 \mathrm{G}$ cellular communications: theoretical feasibility and prototype results. IEEE Communications Magazine, 2014. 52(2): p. 106113.

[69] massive mimo. 2016 [cited 2016 5/8/2016]; Available from: http://5gnews.com/index.php/component/tages/tag/35.

[70] Harris, P., et al., An overview of massive MIMO research at the University of Bristol. 2016.

[71] Marzetta, T.L., Noncooperative cellular wireless with unlimited numbers of base station antennas. IEEE Transactions on Wireless Communications, 2010. 9(11): p. 3590-3600.

[72] Narasimhan, T.L. and A. Chockalingam, Channel hardening-exploiting message passing (CHEMP) receiver in large-scale MIMO systems. IEEE Journal of Selected Topics in Signal Processing, 2014. 8(5): p. 847-860.

[73] Wang, Z., et al. Location-based channel estimation and pilot assignment for massive MIMO systems. in Communication Workshop (ICCW), 2015 IEEE International Conference on. 2015. IEEE.

[74] Prasad, K.S.V., E. Hossain, and V.K. Bhargava, Energy Efficiency in Massive MIMO-Based 5G Networks: Opportunities and Challenges. IEEE Wireless Communications, 2017.

[75] Meerja, K.A. and K. Almustafa, Big Data Outburst due to Wireless Internet of Things. International Journal of Computer Science and Information Security, 2016. 14(5): p. 31.

[76] Kristem, V., et al., 3D MIMO Outdoor-to-Indoor Propagation Channel Measurement. IEEE Transactions on Wireless Communications, 2017.

[77] Mitra, R.N. and D.P. Agrawal, 5G mobile technology: A survey. ICT Express, 2015. 1(3): p. 132-137.

[78] Olsson, M., et al. 5GrEEn: Towards Green 5G mobile networks. in Wireless and Mobile Computing, Networking and Communications (WiMob), 2013 IEEE 9th International Conference on. 2013. IEEE.

[79] Yan, Z., M. Peng, and C. Wang, Economical Energy Efficiency: An Advanced Performance Metric for 5G Systems. IEEE Wireless Communications, 2017. 24(1): p. 32-37.

[80] Taufique, A., et al., Planning Wireless Cellular Networks of Future: Outlook, Challenges and Opportunities. IEEE Access, 2017. 5: p. 48214845.

[81] Bonato, P. Clinical applications of wearable technology. in Engineering in Medicine and Biology Society, 2009. EMBC 2009. Annual International Conference of the IEEE. 2009. IEEE.

[82] Binkley, P.F., Predicting the potential of wearable technology. IEEE engineering in medicine and biology magazine, 2003. 22(3): p. 23-27.

[83] Oleshchuk, V. and R. Fensli, Remote patient monitoring within a future 5G infrastructure. Wireless Personal Communications, 2011. 57(3): p. 431-439.

[84] López, P., et al. Survey of internet of things technologies for clinical environments. in Advanced Information Networking and Applications Workshops (WAINA), 2013 27th International Conference on. 2013. IEEE.

[85] Sodhro, A.H. and M.A. Shah. Role of 5G in medical health. in Innovations in Electrical Engineering and Computational Technologies (ICIEECT), 2017 International Conference on. 2017. IEEE.

[86] NYUSIM, N.Y.U.-. 5G Millimeter Wave (mmW) simulator. 2016 5/8/2016]; Available from: http://wireless.engineering.nyu.edu/5gmillimeter-wave-channel-modeling-software/.

[87] University, B. mmMagic 2016 [cited 2016 5/8/2016]; Available from: http://www.bristol.ac.uk/engineering/research/csn/projects/current/magi $\mathrm{c} /$.

[88] Remcom. InSite. 2016 [cited 2016 5/8/2016]; Available from: http://www.remcom.com/5g-mimo.

[89] SIRADEL 2016 [cited 2016 5/8/2016]; Available from: http://www.siradel.com/software/connectivity/s_5gchannel/.

[90] Mathworks. MATLAB and Simulink 2016 [cited 2016; Available from: http://www.mathworks.com/discovery/5g-wireless-technology.html. 